\title{
Physical Activity of Patients with Chronic Schizophrenia and Related Clinical Factors
}

\author{
Sook-Hyun Lee', Gyurin Kim¹, Chul-Eung $\mathrm{Kim}^{2}$, and Seunghyong Ryu ${ }^{1} \bowtie$ \\ ${ }^{1}$ Department of Mental Health Research, National Center for Mental Health, Seoul, Republic of Korea \\ ${ }^{2}$ Mental Health Research Institute, National Center for Mental Health, Seoul, Republic of Korea
}

Objective This study aimed to investigate clinical factors contributing to the low physical activity (PA) of patients with chronic schizophrenia. Methods PA was measured in 50 outpatients with chronic schizophrenia using the International Physical Activity Questionnaire Short Form (IPAQ-SF). Psychopathology, psychosocial functioning, and extrapyramidal symptoms were assessed using the 18 item-Brief Psychiatric Rating Scale (BPRS-18), Global Assessment of Functioning (GAF), and Drug-Induced Extrapyramidal Symptom Scale (DIEPSS), respectively. We examined differences in these clinical variables between "inactive," "minimally active," and "health enhancing physical activity" groups. Linear regression analysis was used to examine the clinical factors explaining low PA levels in patients with schizophrenia.

Results Subjects spent an average of $130.18 \pm 238.89 \mathrm{~min} /$ wk on moderate/vigorous-intensity PA and only $26 \%$ of them met the recommended PA guideline of 150 minutes of at least moderate PA per week. The inactive group showed significantly higher BPRS-18 and DIEPSS scores, and a lower GAF score than the other groups. Linear regression analysis showed that DIEPSS scores independently explained the amount of total PA ( $\mathrm{p}=0.001)$ and time spent being sedentary $(\mathrm{p}=0.028)$.

Conclusion This study provides preliminary evidence that extrapyramidal symptoms could be a major impediment to the PA of patients with schizophrenia.

Psychiatry Investig 2018;15(8):811-817

Key Words Schizophrenia, Physical activity, Sedentary lifestyle, Extrapyramidal symptoms.

\section{INTRODUCTION}

Patients with schizophrenia have a lower life expectancy owing to higher premature mortality than the general population. ${ }^{1}$ A large cohort study showed that the mortality rate of patients with schizophrenia was 3.7 times higher than that of the general population, and their loss of life expectancy was estimated to be 28.5 years. $^{2}$ According to epidemiological studies, cardiovascular disease is the most common cause of premature mortality in patients with schizophrenia. ${ }^{3,4}$ In particular, low physical activity (PA), sedentary lifestyle, and subsequent obesity and metabolic syndrome are considered major contributors to the high incidence of cardiovascular

Received: November 28, 2017 Revised: April 1, 2018

Accepted: April 15, 2018

$\triangle$ Correspondence: Seunghyong Ryu, MD

Department of Mental Health Research, National Center for Mental Health, 127 Youngmasan-ro, Gwangjin-gu, Seoul 04933, Republic of Korea

Tel: +82-2-2204-0109, Fax: +82-2-2204-0393, E-mail: seunghyongryu@gmail.com

(a) This is an Open Access article distributed under the terms of the Creative Commons Attribution Non-Commercial License (http://creativecommons.org/licenses/bync/4.0) which permits unrestricted non-commercial use, distribution, and reproduction in any medium, provided the original work is properly cited. diseases and premature mortality in patients with schizophrenia. ${ }^{5,6}$

Recent studies suggest that PA can lower the risk of cardiometabolic disease and improve cardiopulmonary fitness, ${ }^{7,8}$ but also alleviate negative symptoms and cognitive decline in patients with schizophrenia. ${ }^{9}{ }^{90}$ However, many patients with schizophrenia suffering from negative symptoms and cognitive impairment are socially withdrawn and less physically active, spending a lot of time sitting or lying down. ${ }^{11,12}$ They often lack the will to improve their health through PA. ${ }^{13,14} \mathrm{In}$ sufficient PA may contribute to an increase in the prevalence of obesity and metabolic syndrome, which are well-known causes of cardiovascular and respiratory diseases, among patients with schizophrenia. ${ }^{15}$ Moreover, limitations in activity due to a decline in cardiopulmonary function results in a vicious cycle in which PA is further reduced. ${ }^{16,17}$ In addition, low PA may exacerbate depressive symptoms, low self-esteem, and impairment of psychosocial functioning in patients with schizophrenia, resulting in lower quality of life. ${ }^{18}$

As life expectancy increases, patients with schizophrenia are more likely to be exposed to chronic physical illnesses 
such as diabetes and cardiovascular diseases. ${ }^{19}$ As a result, social costs may be expected to rise. Considering that adequate PA can reduce the risk of cardiovascular disease and premature mortality, attempts are needed to increase PA in patients with schizophrenia. As mentioned above, PA is also closely related to mental health, including self-esteem, social functioning, and quality of life. In order to improve the physical health of patients with schizophrenia, it is necessary to first examine the clinical factors affecting PA in these patients. The purpose of this study was to investigate PA levels in Korean patients with chronic schizophrenia and to identify the clinical factors related to their PA.

\section{METHODS}

\section{Subjects}

A total of 50 subjects were recruited from an outpatient clinic at the National Center for Mental Health. Inclusion criteria were as follows: 1 ) aged 20-60 years; 2 ) met the Diagnostic and Statistical Manual of Mental Disorders, Fifth Edition (DSM-V) criteria for schizophrenia; 3) received antipsychotic treatment for more than 2 years without major changes in medications; and 4) appeared clinically stable without hospitalization for the past year and could provide reliable information. Patients with other psychiatric disorders, neurological diseases, and physical disability were excluded. This study was initiated after approval from the Institutional Review Board of the National Center for Mental Health (IRB No: 116271-2016-42), and written informed consent was obtained from all subjects.

\section{Assessments}

All subjects completed the Korean version of the International Physical Activity Questionnaire Short Form (IPAQSF), ${ }^{20,21}$ which was developed by the World Health Organization (WHO) for surveillance and global comparison of PA. A trained interviewer (SHL) helped the subjects understand the questionnaire. The 7 items of the IPAQ-SF recorded the total minutes spent on vigorous-intensity $\mathrm{PA}$, moderate-intensity PA, walking, and sedentary behavior (SB) over the 7 days prior to taking the survey. The amount of PA was standardized according to the metabolic equivalent task (MET) minutes per week (MET-min/wk). Using the Ainsworth et al. ${ }^{22}$ compendium, a MET score for each type of activity was estimated by weighting the reported minutes per week by a MET energy expenditure estimate assigned to each type of activity: MET-min/wk=duration $\times$ frequency per week $\times$ MET intensity (vigorous $\mathrm{PA}=8.0 \mathrm{METs}$, moderate $\mathrm{PA}=4.0 \mathrm{METs}$, and walking=3.3 METs). Total PA (total MET-min/wk) was calculated as a sum of vigorous PA, moderate PA, and walk- ing MET-min/wk scores. In addition, body weights and waist circumferences were measured, and body mass indexes (BMI, $\mathrm{kg} / \mathrm{m}^{2}$ ) were calculated.

The clinical assessments were performed by an experienced psychiatrist (SR) using a direct interview and clinicalrating scales. The overall level of psychopathology was evaluated using the 18 item-Brief Psychiatric Rating Scale (BPRS18). ${ }^{23,24}$ The "affect," "positive symptoms," "negative symptoms," "resistance," and "activation" subscales of BPRS-18 were analyzed according to the factor structure proposed by Shafer. ${ }^{25}$ The Global Assessment of Functioning (GAF) was used for assessing the overall level of the patients' functioning. ${ }^{26} \mathrm{Ex}$ trapyramidal symptoms of the patients were assessed using the Korean version of the Drug-Induced Extrapyramidal Symptom Scale (DIEPSS), ${ }^{27,28}$ which was developed to measure drug-induced movement disorders addressing 8 individual items (gait, bradykinesia, sialorrhea, muscle rigidity, tremor, akathisia, dystonia, and dyskinesia) and a global item. Assessments of drug-induced parkinsonism using the DIEPSS were performed by calculating the summed total score of five items, namely gait, bradykinesia, sialorrhea, muscle rigidity, and tremor. ${ }^{28}$

\section{Statistical analyses}

The amount of PA was analyzed using descriptive statistics. We calculated the proportion of the patients meeting the recommended 150 minutes of moderate/vigorous-intensity PA per week for substantial health benefits in patients with schizophrenia according to the International Organization of Physical Therapy in Mental Health (IOPTMH) consensus. ${ }^{29}$ In addition, we measured the mean time of $\mathrm{SB}$ in the patients per day.

We classified the subjects into "inactive," "minimally active," and "health enhancing physically active" groups according to the IPAQ scoring protocol. ${ }^{20}$ Following this, we compared the BPRS-18, GAF, and DIEPSS scores among the 3 groups using the Kruskal-Wallis test, followed by pair-wise comparisons using the Mann-Whitney test, with adjusted $\mathrm{p}$ value of less than 0.017. In addition, we analyzed the subscales of BPRS-18 (affect, positive symptoms, negative symptoms, resistance, and activation) and DIEPSS (parkinsonism, akathisia, and dyskinesia) using the same statistical tests. To explore the clinical factors (BPRS-18, GAF, and DIEPSS scores) that could explain the total PA (total MET-min/wk) or SB in patients with schizophrenia, we performed linear regression analyses, controlling for sex, age, and duration of illness. Total MET-min/wk followed a normal distribution.

All statistical analyses were performed using Predictive Analytics Software (PASW) version 17.0 (SPSS Inc., Chicago, IL, USA). P values of less than 0.05 were considered statisti- 
cally significant, except in the adjusted test for multiple comparisons $(\mathrm{p}<0.017)$.

\section{RESULTS}

\section{Demographic and clinical characteristics}

Table 1 summarizes the demographic characteristics of subjects. A high proportion of the patients were unmarried $(85 \%)$ or unemployed $(82 \%)$. Eighteen $(36 \%)$ patients suffered from one or more of the following: hypertension, diabetes, and hyperlipidemia. More than half (64\%) of the patients had been receiving antipsychotic polypharmacy. Risperidone was the first prescribed antipsychotic drug (34\%), followed by clozapine (30\%), quetiapine (22\%), olanzapine (14\%), paliperidone (14\%), amisulpride (14\%), haloperidol (10\%), blonanserin (8\%), zotepine $(8 \%)$, aripiprazole (6\%), sulpiride (6\%), chlorpromazine (4\%), and ziprasidone (2\%). On average, the patients showed a high level of psychopathology (mean BPRS-18 score $=39.12 \pm 9.08$ ) and a low level of psychosocial functioning (mean GAF score $=49.26 \pm$ 9.48). The mean DIEPSS score was $6.68 \pm 4.32$. There was a weak but significant correlation between the DIEPSS score and antipsychotic dose $(\mathrm{r}=0.36, \mathrm{p}=0.010)$. In addition, $66 \%$ of the patients exceeded the BMI cutoff for obesity in Korea (BMI $\geq 25$ ) and $80 \%$ had abdominal obesity (waist circumference $\geq 90 \mathrm{~cm}$ for men and $\geq 85 \mathrm{~cm}$ for women in Korea).

\section{Physical activity and sedentary behavior of patients with schizophrenia}

Table 2 demonstrates the time patients spent on each type of PA and the amount of PA they engaged in during one week. On average, the patients engaged in $389.74 \pm 401.18$ $\mathrm{min} / \mathrm{wk}$ of total PA, which was equivalent to $1382.07 \pm 1453.40$ MET-min/wk. Walking accounted for about $60 \%$ of the amount of total PA. The patients spent $130.18 \pm 238.89 \mathrm{~min} /$ wk on moderate/vigorous-intensity PA. Only $26 \%$ of them (13/50) met the IOPTMH-recommended PA guideline of 150 minutes of at least moderate-intensity PA per week. The patients were divided into either an inactive group $(n=22)$, minimally active group $(n=20)$, or health enhancing physically active group $(n=8)$ according to the IPAQ scoring protocol. In addition, the patients spent an average of 7.31 \pm 3.07 hours per day being sedentary. There was a significant difference in SB time among inactive, minimally active, and health

Table 1. Demographic and clinical characteristics $(N=50)$

\begin{tabular}{lc}
\hline \multicolumn{1}{c}{ Variable } & Mean \pm SD or N (\%) \\
\hline Age, $\mathrm{y}$ & $47.40 \pm 7.24$ \\
Sex (male/female), N (\%) & $29(58) / 21(42)$ \\
Marital status, N (\%) & $36(72) / 7(14) / 7(14)$ \\
$\quad$ Single/divorced/married & $16(32)$ \\
Education beyond high school, N (\%) & $2(4) / 7(14) / 0(0) / 41(82)$ \\
Employment status, N (\%) & $8(16) / 15(30) / 6(12)$ \\
$\quad$ Employed/housewife/student/unemployed & \\
Comorbidity, N (\%) & $33(66) / 3(6) / 14(28)$ \\
$\quad$ Hypertension/DM/hyperlipidemia & $25.98 \pm 8.46$ \\
Smoking history, N (\%) & $21.32 \pm 7.94$ \\
$\quad$ Non-smoker/quit smoking/smoker & $866.65 \pm 461.70$ \\
Onset age, y & \\
Duration of illness, $y$ & \\
Current antipsychotic dose (chlorpromazine equivalents), mg &
\end{tabular}

Table 2. Mean amount of physical activity in patients with chronic schizophrenia

\begin{tabular}{lccc}
\hline & Time $(\mathrm{min} / \mathrm{wk})$ & MET-min/wk & Ratio of MET (\%) \\
\hline Walking & $259.56 \pm 292.22$ & $856.55 \pm 964.31$ & 61.98 \\
Moderate PA & $128.98 \pm 238.32$ & $515.92 \pm 953.29$ & 37.33 \\
Vigorous PA & $1.20 \pm 8.49$ & $9.60 \pm 67.88$ & 0.69 \\
Total PA & $389.74 \pm 401.18$ & $1382.07 \pm 1453.40$ & 100 \\
\hline
\end{tabular}

MET-min/wk: metabolic equivalent task-minutes per week, PA: physical activity 
enhancing physically active groups $\left(\chi^{2}=23.53, \mathrm{p}<0.001\right)$; patients in the inactive group engaged in more SB than those in minimal active group $(U=70.00, p<0.001)$ or health enhancing physically active group $(\mathrm{U}=0.00, \mathrm{p}<0.001)$.

\section{Association between physical activity and clinical factors in patients with schizophrenia}

We found significant differences in BPRS-18 $\left(\chi^{2}=16.98, \mathrm{p}<\right.$ $0.001), \operatorname{GAF}\left(\chi^{2}=16.49, \mathrm{p}<0.001\right)$, and DIEPSS scores $\left(\chi^{2}=24.82\right.$, $\mathrm{p}<0.001)$ among inactive, minimally active, and health enhancing physically active groups (Table 3). Pair-wise comparisons confirmed that BPRS and DIEPSS scores in the inactive group were significantly higher than those in the minimally active group (BPRS: $U=78.50, p<0.001$; DIEPSS: $\mathrm{U}=71.00, \mathrm{p}<0.001)$ or health enhancing physically active group (BPRS: $\mathrm{U}=22.00, \mathrm{p}=0.001$; DIEPSS: $\mathrm{U}=1.50, \mathrm{p}<0.001$ ). Specifically, physically inactive patients suffered from a significantly higher level of affective, positive, and negative symptoms as well as drug-induced parkinsonism than minimally active (affect: $\mathrm{U}=106.00, \mathrm{p}=0.004$; negative: $\mathrm{U}=113.00$, $\mathrm{p}=0.006$; parkinsonism: $U=75.00, \mathrm{p}<0.001)$ or health enhancing physically active patients (positive: $\mathrm{U}=33.00, \mathrm{p}=$ 0.008; negative: $U=7.00, p<0.001$; parkinsonism: $U=3.50$, $\mathrm{p}<0.001)$. In addition, the inactive group also showed a significantly lower GAF score than minimally active $(U=107.50$, $\mathrm{p}=0.004)$ and health enhancing physically active $(U=16.00$, $\mathrm{p}<0.001)$ groups. Linear regression analysis showed that the DIEPSS score independently explained the amount of physical activity $(\mathrm{B}=-264.88, \mathrm{t}=-3.60, \mathrm{p}=0.001)$ and $\mathrm{SB}$ time $(\mathrm{B}=0.35, \mathrm{t}=2.27, \mathrm{p}=0.028)$ (Table 4$)$.

\section{DISCUSSION}

In this study, we assessed the PA levels of patients with schizophrenia using the IPAQ-SF and identified clinical factors associated with their PA. To the best of our knowledge, this is the first study to investigate the PA of outpatients with chronic schizophrenia in Korea. We found that over $70 \%$ of patients with schizophrenia did not meet the recommended PA guideline of 150 minutes of moderate/vigorous-intensity PA per week. About half of the patients in this study were classified as inactive according to the IPAQ scoring protocol, and they showed higher levels of psychopathology and EPS, and lower levels of psychosocial functioning than physically active patients. In particular, we observed that the level of EPS independently explained the amount of total PA in patients with schizophrenia.

We observed that Korean patients with chronic schizophrenia spent about $400 \mathrm{~min} /$ wk engaging in PA of about 1400 min-MET/wk. This is comparable to the result of a Belgian study in which patients with schizophrenia spent about 330 $\mathrm{min} /$ wk engaging in PA, which was equivalent to about 1300 min-MET/wk. ${ }^{8}$ However, considering that the mean amount of PA in the general population was about $3000 \mathrm{~min}-\mathrm{MET} /$ wk in the validation study of Korean version of IPAQ-SF, ${ }^{21}$ it is clear that patients with schizophrenia are less physically active than the general population in Korea. Furthermore, patients with schizophrenia spent about $130 \mathrm{~min} /$ wk engaging in moderate/vigorous-intensity PA. Only $26 \%$ of them met the IOPTMH-recommended PA guideline for minimizing cardio-metabolic risk in patients with schizophrenia of 150 minutes of at least moderate-intensity PA per week. This pro-

Table 3. Association between physical activity and clinical factors in chronic patients with schizophrenia

\begin{tabular}{lcccc}
\hline & Inactive $(\mathrm{N}=22)$ & Minimally active $(\mathrm{N}=20)$ & Health enhancing $(\mathrm{N}=8)$ & Statistics $^{\dagger}$ \\
\hline BPRS-18 & $44.86 \pm 8.52$ & $35.60 \pm 7.15^{*}$ & $32.13 \pm 5.03^{*}$ & $\chi^{2}=16.98, \mathrm{p}<0.001$ \\
Affect & $9.27 \pm 2.59$ & $6.80 \pm 2.46^{*}$ & $7.00 \pm 1.77$ & $\chi^{2}=10.32, \mathrm{p}=0.006$ \\
Positive & $11.55 \pm 3.54$ & $9.55 \pm 2.86$ & $7.50 \pm 3.12^{*}$ & $\chi^{2}=8.96, \mathrm{p}=0.011$ \\
Negative & $12.36 \pm 1.97$ & $9.75 \pm 3.06^{*}$ & $8.00 \pm 1.31^{*}$ & $\chi^{2}=18.06, \mathrm{p}<0.001$ \\
Resistance & $5.48 \pm 1.97$ & $4.50 \pm 1.70$ & $4.38 \pm 1.30$ & $\chi^{2}=3.84, \mathrm{p}=0.147$ \\
Activation & $5.18 \pm 1.71$ & $4.00 \pm 1.17$ & $4.25 \pm 0.89$ & $\chi^{2}=6.30, \mathrm{p}=0.043$ \\
GAF & $44.00 \pm 9.72$ & $51.35 \pm 6.85^{*}$ & $58.50 \pm 4.69^{*}$ & $\chi^{2}=16.49, \mathrm{p}<0.001$ \\
DIEPSS ${ }^{*}$ & $9.91 \pm 2.84$ & $5.10 \pm 3.70^{*}$ & $1.75 \pm 1.28^{*}$ & $\chi^{2}=24.82, \mathrm{p}<0.001$ \\
Parkinsonism & $6.95 \pm 1.86$ & $3.70 \pm 2.62^{*}$ & $1.38 \pm 1.19^{*}$ & $\chi^{2}=23.81, \mathrm{p}<0.001$ \\
Akathisia & $0.50 \pm 0.80$ & $0.25 \pm 0.64$ & $0.25 \pm 0.46$ & $\chi^{2}=1.57, \mathrm{p}=0.456$ \\
Dyskinesia & $0.36 \pm 0.73$ & $0.10 \pm 0.31$ & $0.00 \pm 0.00$ & $\chi^{2}=4.15, \mathrm{p}=0.126$ \\
\hline
\end{tabular}

${ }^{*} \mathrm{p}<0.017$ comparing inactive and minimally active groups or comparing inactive and health enhancing groups using Mann-Whitney test, †Kruskal-Wallis test, ‡total score and subscale scores according to the factor structure proposed by Shafer ${ }^{25}$, \$total score and subscale scores. Parkinsonism score is the summed score of 5 items for gait, bradykinesia, sialorrhea, muscle rigidity, and tremor. ${ }^{28}$ BPRS-18: 18 item-Brief Psychiatric Rating Scale, GAF: Global Assessment of Functioning, DIEPSS: Drug-Induced Extrapyramidal Symptom Scale 
Table 4. Clinical factors contributing to physical activity and sedentary behaviora*

\begin{tabular}{|c|c|c|c|c|}
\hline & $\mathrm{B}$ & SE & $\mathrm{t}$ & $\mathrm{P}$ \\
\hline \multicolumn{5}{|c|}{ Total physical activity (total MET-min/wk) ${ }^{\dagger}$} \\
\hline Sex & -109.06 & 328.75 & -0.33 & 0.742 \\
\hline Age & 10.44 & 24.37 & 0.43 & 0.670 \\
\hline Duration of illness & -5.98 & 24.90 & -0.24 & 0.811 \\
\hline BPRS-18 & 49.42 & 26.54 & 1.86 & 0.069 \\
\hline GAF & 20.39 & 18.46 & 1.11 & 0.275 \\
\hline DIEPSS & -264.88 & 73.68 & -3.60 & 0.001 \\
\hline \multicolumn{5}{|l|}{ Sedentary behavior $\ddagger$} \\
\hline Sex & 0.91 & 0.68 & 1.35 & 0.186 \\
\hline Age & 0.01 & 0.05 & 0.21 & 0.838 \\
\hline Duration of illness & -0.04 & 0.05 & -0.69 & 0.495 \\
\hline BPRS-18 & 0.09 & 0.06 & 1.69 & 0.099 \\
\hline GAF & 0.01 & 0.04 & 0.17 & 0.866 \\
\hline DIEPSS & 0.35 & 0.15 & 2.27 & 0.028 \\
\hline
\end{tabular}

*to explore what kind of clinical factors explains total physical activity or sedentary behavior, we performed linear regression analyses, controlling for sex, age, and duration of illness, ${ }^{\dagger} \mathrm{R}^{2}=0.71,{ }^{\ddagger} \mathrm{R}^{2}=0.92$. MET-min/wk: metabolic equivalent task-minutes per week, BPRS-18: 18 item-Brief Psychiatric Rating Scale, GAF: Global Assessment of Functioning, DIEPSS: Drug-Induced Extrapyramidal Symptom Scale

portion is very low compared to the results of a meta-analysis conducted by Stubbs et al., ${ }^{11}$ in which $56.6 \%$ of patients with schizophrenia met the PA guideline, and suggests that the majority of patients with schizophrenia in Korea have not engaged in PA sufficient to improve their physical health.

Sedentary behavior, defined as an energy expenditure $\leq 1.5$ METs while in a sitting or reclining posture during waking hours, ${ }^{30,31}$ is also known to be an independent risk factor for cardiovascular disease and mortality. ${ }^{32}$ In this study, Korean patients with schizophrenia reported that they spent on average 7.31 hours per day being sedentary. A meta-analysis study of SB in patients with psychosis reported that the mean time of self-reported SB was 6.85 hours per day, ${ }^{12}$ which is comparable to our findings. However, in the meta-analysis, the duration of SB increased to 12.6 hour per day when measured via objective measures such as an accelerometer. ${ }^{12}$ In this regard, the IPAQ-SF may underestimate the time spent being sedentary in Korean patients with schizophrenia. Research on the general population demonstrated that engaging in SB for more than 3 hours per day was associated with a reduction in life expectancy by 2 years. ${ }^{33}$ Therefore, reducing SB of patients with schizophrenia will be critical for lowering the rate of their premature mortality.

Some clinical factors might influence the PA of patients with schizophrenia. For example, social withdrawal due to positive or negative symptoms may lead to a decrease in PA of patients with schizophrenia. ${ }^{34,35}$ Unemployment or social isolation due to low social functioning in patients with schizophrenia may also reduce their PA and increase the amount of time they spend being sedentary. ${ }^{36}$ In addition, deterioration of physical fitness due to antipsychotic-induced EPS or weight gain may be an important factor hindering PA of patients with schizophrenia. ${ }^{8,37}$ In this study, we observed that physically inactive patients suffered from a higher level of psychopathology and a lower level of social functioning than minimally active or health enhancing physically active patients. In particular, our findings suggested that factors contributing to physical inactivity in patients with schizophrenia encompass not only passive social withdrawal due to negative symptoms or low social functioning, but also active social avoidance due to residual positive or affective symptoms. In addition, physically inactive patients showed a higher level of drug-induced parkinsonism than minimally active or health enhancing physically active patients. Moreover, we found that the level of EPS to be an independent explanatory factor for the amount of total PA and time spent being sedentary in patients with schizophrenia. Patients with chronic schizophrenia are likely to have many risk factors for EPS. ${ }^{38}$ In this study, many of the subjects were middle-aged and had been receiving high-dose antipsychotic polypharmacy. Their daily dose of antipsychotics was also significantly correlated with the level of EPS. Therefore, we need to recognize that EPS is a major impediment to the PA of patients with schizophrenia and should be monitored more carefully. In summary, our findings show that PA levels of patients with schizophrenia are associated with their levels of psychopathology, social functioning, and EPS. Effective treatment of residual symptoms and EPS, as well as social rehabilitation, will be 
crucial for improving the PA and physical health of patients with schizophrenia.

This study has some methodological limitations. First, the reliance on self-reported recall of PA is an important limitation. Patients with schizophrenia may overestimate their PA level and underestimate SB time because they are considerably less active than the general population and may have difficulties in accurately recalling their PA intensity and SB time. Second, this study included many patients with relatively high levels of psychopathology and chronicity. Therefore, careful attention should be paid to interpretation of our findings as a representative result of the entire population of patients with schizophrenia. Third, because this study did not include healthy controls, we could not directly compare PA levels between patients with schizophrenia and healthy controls matched for age, sex, and BMI. Fourth, this study did not examine the laboratory markers of metabolic syndrome. As such, we could not investigate the association between PA and metabolic syndrome in patients with schizophrenia.

Taking these limitations into account, this study suggests that a large number of patients with schizophrenia face the risk of health deterioration due to lack of PA and attempts are needed to improve correctable clinical factors, such as EPS, as much as possible for the promotion of PA and prevention of SB. Future studies evaluating the PA of patients with schizophrenia and its related factors with objective measures, and a larger sample size are warranted.

\section{Acknowledgments}

This work was supported by a clinical research grant (code 2017-11) from the National Center for Mental Health, Republic of Korea.

\section{REFERENCES}

1. Walker ER, McGee RE, Druss BG. Mortality in mental disorders and global disease burden implications: a systematic review and metaanalysis. JAMA Psychiatry 2015;72:334-341.

2. Olfson M, Gerhard T, Huang C, Crystal S, Stroup TS. Premature mortality among adults with schizophrenia in the United States. JAMA Psychiatry 2015;72:1172-1181.

3. Bresee LC, Majumdar SR, Patten SB, Johnson JA. Prevalence of cardiovascular risk factors and disease in people with schizophrenia: a population-based study. Schizophr Res 2010;117:75-82.

4. Saha S, Chant D, McGrath J. A systematic review of mortality in schizophrenia: is the differential mortality gap worsening over time? Arch Gen Psychiatry 2007;64:1123-1131.

5. Brown S, Birtwistle J, Roe L, Thompson C. The unhealthy lifestyle of people with schizophrenia. Psychol Med 1999;29:697-701.

6. Osborn DP, Nazareth I, King MB. Physical activity, dietary habits and Coronary Heart Disease risk factor knowledge amongst people with severe mental illness: a cross sectional comparative study in primary care. Soc Psychiatry Psychiatr Epidemiol 2007;42:787-793.

7. Firth J, Cotter J, Elliott R, French P, Yung AR. A systematic review and meta-analysis of exercise interventions in schizophrenia patients. Psychol Med 2015;45:1343-1361.

8. Vancampfort D, Probst M, Scheewe T, De Herdt A, Sweers K, Knapen J, et al. Relationships between physical fitness, physical activity, smok- ing and metabolic and mental health parameters in people with schizophrenia. Psychiatry Res 2013;207:25-32.

9. Dauwan M, Begemann MJ, Heringa SM, Sommer IE. Exercise improves clinical symptoms, quality of life, global functioning, and depression in schizophrenia: a systematic review and meta-analysis. Schizophr Bull 2016;42:588-599.

10. Kimhy D, Lauriola V, Bartels MN, Armstrong HF, Vakhrusheva J, Ballon JS, et al. Aerobic exercise for cognitive deficits in schizophrenia the impact of frequency, duration, and fidelity with target training intensity. Schizophr Res 2016;172:213-215.

11. Stubbs B, Firth J, Berry A, Schuch FB, Rosenbaum S, Gaughran F, et al. How much physical activity do people with schizophrenia engage in? A systematic review, comparative meta-analysis and meta-regression. Schizophr Res 2016;176:431-440.

12. Stubbs B, Williams J, Gaughran F, Craig T. How sedentary are people with psychosis? A systematic review and meta-analysis. Schizophr Res 2016;171:103-109.

13. Vancampfort D, De Hert M, Stubbs B, Ward PB, Rosenbaum S, Soundy A, et al. Negative symptoms are associated with lower autonomous motivation towards physical activity in people with schizophrenia. Compr Psychiatry 2015;56:128-132.

14. Vancampfort D, De Hert M, Vansteenkiste M, De Herdt A, Scheewe TW, Soundy A, et al. The importance of self-determined motivation towards physical activity in patients with schizophrenia. Psychiatry Res 2013;210:812-818.

15. Vancampfort D, Probst M, Knapen J, Carraro A, De Hert M. Associations between sedentary behaviour and metabolic parameters in patients with schizophrenia. Psychiatry Res 2012;200:73-78.

16. Vancampfort D, Probst M, Stubbs B, Soundy A, De Herdt A, De Hert M. Metabolic syndrome and lung function in schizophrenia: a pilot study. Psychiatry Res 2014;220:58-62.

17. Vancampfort D, Probst M, Stubbs B, Soundy A, De Herdt A, De Hert $\mathrm{M}$. Associations between expiratory spirometry parameters and limitations in daily life activities in patients with schizophrenia. Gen Hosp Psychiatry 2014;36:172-176.

18. Vancampfort D, Probst M, Scheewe T, Maurissen K, Sweers K, Knapen J, et al. Lack of physical activity during leisure time contributes to an impaired health related quality of life in patients with schizophrenia. Schizophr Res 2011;129:122-127.

19. Smith DJ, Langan J, McLean G, Guthrie B, Mercer SW. Schizophrenia is associated with excess multiple physical-health comorbidities but low levels of recorded cardiovascular disease in primary care: crosssectional study. BMJ Open 2013;3.pii: e002808.

20. Craig CL, Marshall AL, Sjostrom M, Bauman AE, Booth ML, Ainsworth BE, et al. International physical activity questionnaire: 12-country reliability and validity. Med Sci Sports Exerc 2003;35:1381-1395.

21. Oh JY, Yang YJ, Kim BS, Kang JH. Validity and reliability of Korean version of International Physical Activity Questionnaire (IPAQ) short form. Korean J Fam Med 2007;28:532-541.

22. Ainsworth BE, Haskell WL, Whitt MC, Irwin ML, Swartz AM, Strath SJ, et al. Compendium of physical activities: an update of activity codes and MET intensities. Med Sci Sports Exerc 2000;32:S498-S504.

23. Overall JE, Gorham DR. The brief psychiatric rating scale. Psychol Rep 1962;10:799-812.

24. Overall JE, Hollister LE, Pichot P. Major psychiatric disorders. A fourdimensional model. Arch Gen Psychiatry 1967;16:146-151.

25. Shafer A. Meta-analysis of the brief psychiatric rating scale factor structure. Psychol Assess 2005;17:324-335.

26. Jones SH, Thornicroft G, Coffey M, Dunn G. A brief mental health outcome scale-reliability and validity of the Global Assessment of Functioning (GAF). Br J Psychiatry 1995;166:654-659.

27. Inada T. Evaluation and Diagnosis of Drug-Induced Extrapyramidal Symptoms: Commentary on the DIEPSS and Guide to Its Usage. Tokyo: Seiwa Shoten Publishers; 1996.

28. Kim JH, Jung HY, Kang UG, Jeong SH, Ahn YM, Byun HJ, et al. Met- 
ric characteristics of the drug-induced extrapyramidal symptoms scale (DIEPSS): a practical combined rating scale for drug-induced movement disorders. Mov Disord 2002;17:1354-1359.

29. Vancampfort D, De Hert M, Skjerven LH, Gyllensten AL, Parker A, Mulders N, et al. International Organization of Physical Therapy in Mental Health consensus on physical activity within multidisciplinary rehabilitation programmes for minimising cardio-metabolic risk in patients with schizophrenia. Disabil Rehabil 2012;34:1-12.

30. Owen N, Sugiyama T, Eakin EE, Gardiner PA, Tremblay MS, Sallis JF. Adults' sedentary behavior determinants and interventions. Am J Prev Med 2011;41:189-196.

31. Pate RR, O'Neill JR, Lobelo F. The evolving definition of "sedentary". Exerc Sport Sci Rev 2008;36:173-178.

32. Biswas A, Oh PI, Faulkner GE, Bajaj RR, Silver MA, Mitchell MS, et al. Sedentary time and its association with risk for disease incidence, mortality, and hospitalization in adults: a systematic review and metaanalysis. Ann Intern Med 2015;162:123-132.

33. Katzmarzyk PT, Lee IM. Sedentary behaviour and life expectancy in the USA: a cause-deleted life table analysis. BMJ Open 2012;2.pii: e000828.
34. Vancampfort D, Knapen J, Probst M, Scheewe T, Remans S, De Hert M. A systematic review of correlates of physical activity in patients with schizophrenia. Acta Psychiatr Scand 2012;125:352-362.

35. Shin S, Yeom CW, Shin C, Shin JH, Jeong JH, Shin JU, et al. Activity monitoring using a mHealth device and correlations with psychopathology in patients with chronic schizophrenia. Psychiatry Res 2016; 246:712-718.

36. Soundy A, Freeman P, Stubbs B, Probst M, Coffee P, Vancampfort D. The transcending benefits of physical activity for individuals with schizophrenia: a systematic review and meta-ethnography. Psychiatry Res 2014;220:11-19.

37. Vancampfort D, Probst M, Daenen A, Damme TV, De Hert M, Rosenbaum S, et al. Impact of antipsychotic medication on physical activity and physical fitness in adolescents: An exploratory study. Psychiatry Res 2016;242:192-197.

38. Gao K, Kemp DE, Ganocy SJ, Gajwani P, Xia G, Calabrese JR. Antipsychotic-induced extrapyramidal side effects in bipolar disorder and schizophrenia: a systematic review. J Clin Psychopharmacol 2008;28: 203-209. 